\title{
Quantification of age-related differences in reaching and circle-drawing using a robotic rehabilitation device
}

\author{
Alessandra Pacilli ${ }^{\mathrm{a}, \mathrm{b}, 1}$, Marco Germanotta ${ }^{\mathrm{a}, \mathrm{b}, 1}$, Stefano Rossi ${ }^{\mathrm{b}, \mathrm{c}, *}$ and Paolo Cappa ${ }^{\mathrm{a}, \mathrm{b}}$ \\ a DIMA, Department of Mechanical and Aerospace Engineering, "Sapienza" University of Rome, Rome, Italy \\ b MARLab, Movement Analysis and Robotics Laboratory, Neurorehabilitation Division, IRCCS Children's Hospital \\ "Bambino Gesù”, Rome, Italy \\ ${ }^{c}$ DEIM, Department of Economics and Management - Industrial Engineering, University of Tuscia, Viterbo, Italy
}

\begin{abstract}
.
BACKGROUND: Although robotic therapy is at the forefront of upper limb rehabilitation, there is limited information about the importance of selecting age-matched subjects to evaluate recovery of arm movement during rehabilitation.

OBJECTIVE: This study aims to quantify differences in the arm motion of healthy children and adults when they interact with a planar robot, in order to determine whether an age-matched control group is necessary in clinical studies involving pediatric patients.

METHODS: Ten children (aged 7 to 10 years) and ten adults (aged 23 to 25 years) performed, at self-selected speed and accuracy, planar-reaching and circle-drawing movements with a robotic device. We analyzed the motor performances for the two groups quantifying the participants' dexterity in completing two chosen tasks. The measurement of the entire upper limb was obtained by merging the data provided by the robot with that of an optical tracking system.

RESULTS: Children drew circles with less smoothness than adults but with the same accuracy and joint coordination. During planar reaching task, children optimized only the coordination but performed the movement with less accuracy and smoothness than adults.

CONCLUSIONS: Our findings provide evidence that age-matched healthy children should be used to quantify the recovery of robot-mediated therapy in children with upper limb impairments.
\end{abstract}

Keywords: Age-related differences, smoothness, upper limb motor control, robot-mediated therapy, reaching, tracking

\section{Introduction}

During the 1990s [51] robot-mediated therapy (RMT) emerged as an effective tool to restore the motor function of the upper limb [29, 30, 38, 39], both in stroke survivors $[10,11,13,32,37]$ and, more

\footnotetext{
${ }^{1}$ These authors contributed equally to this work.

*Corresponding author: Stefano Rossi, Department of Economics and Management - Industrial Engineering, University of Tuscia, Viterbo, Italy. Tel.: +39 0761 357049; E-mail: stefano.rossi@unitus.it.
}

recently, in children with cerebral palsy $[4,12,16,17$, 53].

In order to assess the residual motor ability of impaired subjects and to quantify the effects of RMT on patients, the collection of baseline data from a control group of healthy subjects is generally needed [23, 27, $31,35]$. Thus, the question of whether the control group has to be age-matched or not is crucial in experimental design. Yadav and colleagues [56] have demonstrated that young and mature healthy subjects respond differently while interacting with a planar rehabilitation 
robot and, consequently, they highlighted the importance of a similar control group in studies with mature stroke survivors. No studies, to the best of our knowledge, have been conducted to ascertain whether the control group has to be age-matched in RMT clinical studies involving pediatric patients.

A number of developmental studies indicated that the complete development of motor control is achieved at different ages as a function of: (i) the required motor tasks, such as reaching or drawing; (ii) the plane where the arm movement is performed; and (iii) the number of degrees of freedom that has to be coordinated in order to complete the motor task. However, the examination of studies based on the evaluation of motor behavior of healthy children and adults while performing goaldirected [36, 44, 48, 49] and drawing [40, 41] tasks without a rehabilitation robot can only partially address our question. It is important to note that the two tasks are commonly used in RMT [3,25]. Studies focused on goal-directed tasks have reported that a younger cohort, as compared with adults, demonstrates differences in arm movements that can be summarized as slower movements, and lower smoothness and accuracy [36, $48,49]$ together with an increase in the variability of results in arm movements [44, 49]. In particular, Thomas et al. [48] reported that children showed lower speed and accuracy than adults while performing rapid goal-directed tasks in a frontal plane. Scheneiberg et al. [44] found that children aged 6 to 8 years had motor performance similar to adults in trajectory kinematics when performing movements in the sagittal plane, while an increased variability in inter-joint coordination patterns persisted also in 11-year-old children. Conversely, Traynor et al. [49] found that during repetitive reaching, data variability increased and movement smoothness decreased comparing adults with children; they concluded that the neuromuscular strategies of children do not match an adult-like pattern. Regarding drawing tasks, Robertson [40, 41] found that children aged 8 to 10 years performed the required task in a manner similar to adults.

Due to the different level of motor control development in upper arm movement, the evaluation of differences between children and adults when they execute typical RMT movements is required to assess whether the control subjects have to be from the same age group. Few studies have been conducted in order to highlight differences in the motor adaptation of children and adults to force-dependent fields in reaching movements performed with a robotic device $[21,22$,
46]. While Jansen-Osmann et al. [21] and Konczak et al. [22] used an ad-hoc robotic device, Takahashi et al. used a commercially available robotic device (Phantom, Sensable Technologies, Inc., Woburn, MA), which is generally utilized to quantify the RMT efficacy [2]. Takahashi et al. [46] observed that, in performing uninhibited movements, children's hands had greater trial-to-trial variability than adults' hands. However, these findings cannot completely characterize the upper arm age-related differences, since they were limited to hand kinematics and kinetics and to reaching movements performed only in one direction.

Thus, to quantify the age-related differences between adults and children with a wider focus, our research chose: (i) to use the 2 DOFs, the planar module of the MIT-Manus, since it is one of the most recognized rehabilitation robotic devices [28]; (ii) to analyze the motor tasks that are commonly used during robotic rehabilitation, such as point-to-point reaching movements and circle-drawing tasks.

In conclusion, this study is designed to assess whether the control subjects have to be within the same age group in RMT clinical studies involving pediatric subjects, by answering the following question: Are movement coordination, accuracy and smoothness significantly different between children and adults when they perform circle-drawing and point-to-point tasks with a rehabilitation robot? The results can provide new insight into the developmental aspects of motor control that can be useful in the design of controllers for robotic rehabilitation devices.

\section{Methods}

\subsection{Ethics statement}

Informed consent, in written form, was obtained from all adults and parents or legal guardians of the children involved in the study. The Research Ethics and Medical Board of the Bambino Gesù Children's Hospital approved the experimental protocol. The protocol conforms to the ethical standards outlined in the 1964 Declaration of Helsinki.

\subsection{Subjects}

A convenience sample of ten normally developed, unimpaired, right-hand-dominant children (age range: 7 to 10 years, body mass: $36.4 \pm 4.7 \mathrm{~kg}$, height: 
$140 \pm 7 \mathrm{~cm})$ and ten healthy, unimpaired, right-handdominant young adults (age range: 23 to 25 years, body mass: $67.3 \pm 9.7 \mathrm{~kg}$, height: $165 \pm 10 \mathrm{~cm}$ ) was enrolled in this study at the Movement Analysis and Robotics Laboratory (MARlab) of the Bambino Gesù Children's Hospital. Hand dominance was established as the hand that participants used for writing and personal activities.

The inclusion criteria for all subjects were: absence of neurological and visual deficits, physiological range of motion (ROM) for elbow and shoulder, and adequate anthropometric measures in order to freely move the end-effector in the robot workspace. All subjects were naive to the robotic device and the tasks.

\subsection{Equipment}

The comparative examination of upper arm movements in children and young adults was performed via a system composed of a robotic device and an optical tracking system.

We used the InMotion Arm Robot (Interactive Motion Technologies Inc., Watertown - USA), which is the commercial version of the MIT-Manus, designed for the rehabilitation of elbow and shoulder. This robot, widely tested on adults, has already been used in studies with children $[12,17,24]$. Therefore, the highly backdriveable low-friction robot does not interfere with motion and allows the individual to freely move the end-effector. In addition it provides two translational degrees of freedom (DOF) and restricts the subject's hand motion to the horizontal plane. During all movements analyzed in this paper, the robot acted as a low friction passive measurement device. The machine records at $200 \mathrm{~Hz}$ the end-effector position (with accuracy of $0.1 \mathrm{~mm}$ ) and force (with resolution of $0.025 \mathrm{~N}$ for $\mathrm{F}_{\mathrm{x}}$ and $\mathrm{F}_{\mathrm{y}}, 0.05 \mathrm{~N}$ for $\mathrm{F}_{\mathrm{z}}$, and 0.00125 $\mathrm{Nm}$ for $\mathrm{T}_{\mathrm{x}}, \mathrm{T}_{\mathrm{y}}$ and $\mathrm{T}_{\mathrm{z}}$ ). A computer screen located in front of the participant provided visual feedback displaying in real-time the location of the desired targets and of the handle's position.

A six cameras optical tracking system (Vicon 512 camera-workstation, workstation 4.0 software, $120 \mathrm{~Hz}$, Oxford UK) was used. Static and dynamic calibration procedures, performed in accordance with the manufacturer's indications, showed that the overall RMS error associated with the marker position was $\sim 1 \mathrm{~mm}$, over a calibration volume of $1 \mathrm{~m} \times 1 \mathrm{~m} \times 0.5 \mathrm{~m}$ centered on the robot workspace.

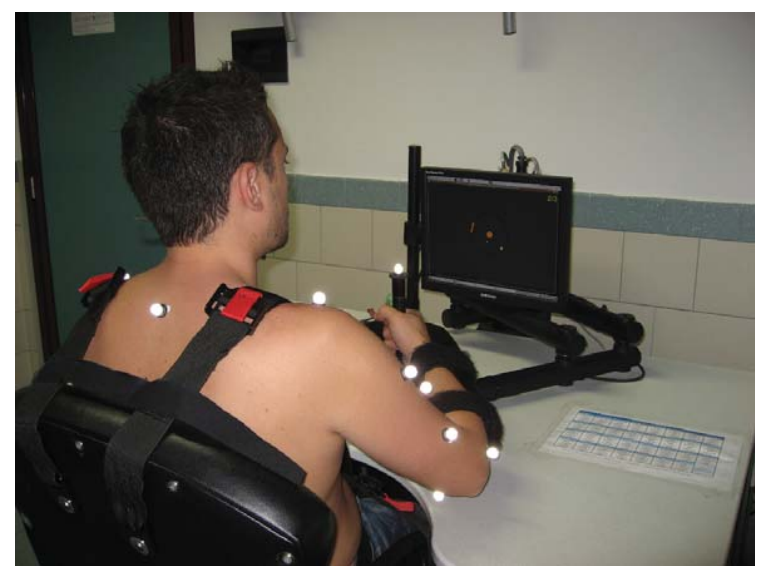

Fig. 1. Young adult participant engaged in the circle-drawing task with InMotion Arm Robot installed at the Movement Analysis and Robotics Laboratory (MARlab) of the Bambino Gesù Children's Hospital. Except for the one placed on the sternum, all the markers for the optical tracker system are showed in the figure.

Data provided by the robot were low-pass filtered and then resampled at $120 \mathrm{~Hz}$ and smoothed by using a 6th order Butterworth filter (cut-off frequency: $10 \mathrm{~Hz}$ ) while marker positions were postprocessed via the Woltring filter (Mean Square Error: 30).

\subsection{Procedure}

Participants were comfortably seated on a chair in front of a table, whereon the video screen was placed at a distance of $55 \mathrm{~cm}$, with their dominant hand grasping the end-effector, see Fig. 1. Since the end-effector diameter was $3.0 \mathrm{~cm}$, both the adults and the children were able to grasp it comfortably. A five-point seatbelt, with vertical straps positioned anterior-medially to the shoulders, was applied during all sessions to limit trunk compensation movements. In addition the right participants' forearm was strapped to a support to limit/prevent all wrist movements and forearm pronosupination. The center of the workspace was located in front of the subject at the body midline. As regards the initial position, for each subject the chair was positioned so that, with the end-effector located on the central target, the elevation of the shoulder was $45^{\circ}$ and the elbow was slightly flexed. As the chair was blocked with respect to the table, we asked the children to move the end-effector in order to check if they could comfortably reach all workspace points. Each of the children enrolled in this study was able to do that. 

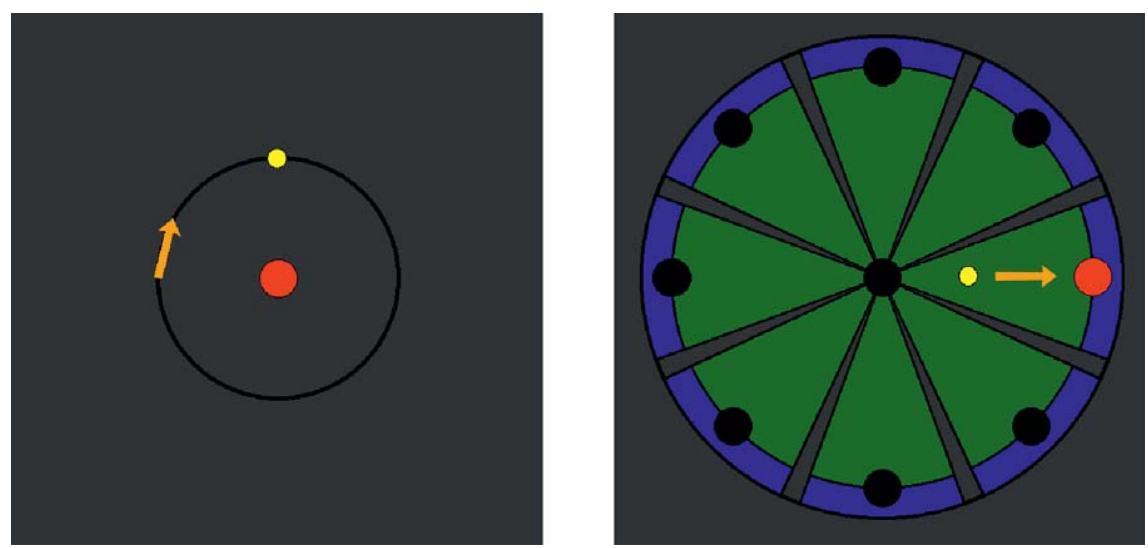

Fig. 2. Visual template of: (left panel) circle-drawing task and (right panel) point-to-point tasks. For both tasks, the yellow circle indicates the position of the end-effector. In the circle-drawing task, the arrow indicates starting point and direction (CW in the figure). In the point-to-point task, the target to be reached is showed by the red circle. (Colours are visible in the online version of the article; http://dx.doi.org/10.3233/ABB-140095)

Nine markers for the optical tracking system were placed over subjects' specific anatomic landmarks: 4 markers on the trunk (sternum, left and right acromion, and 7 th cervical vertebra), 3 not collinear on the upper arm, and 2 on the elbow (medial and lateral epicondile). An additional marker was placed on top of the robot end-effector and was also used to synchronize the signals gathered by the two systems.

The testing protocol consisted of: (i) an unassisted tracking task (circle-drawing); and (ii) an unassisted planar reaching task (point-to-point). The selected tasks are generally used in the rehabilitation treatment delivered by commercially available systems as InMotion Arm Robot [24], Gentle [1] or ad-hoc research prototypes [45]. Every participant took part in both tasks and no specific instructions were given; consequently, velocity and accuracy were self-selected. Verbal encouragement and environmental distractions were kept to a minimum.

In the circle-drawing task a circular template of $8 \mathrm{~cm}$ radius was displayed on the screen (Fig. 2-left), and participants were instructed to "trace the circle" moving the end-effector along the circumference. Their hand was initially positioned at " 9 o' clock" and the participants were asked to draw in sequence five clockwise circles (CW) and five counterclockwise circles (CCW) starting and ending at the same point.

In the point-to-point task, eight targets equally spaced in a circular pattern (radius: $14 \mathrm{~cm}$ ) and one target positioned in the circumference center, coincident with the workspace center, were displayed on the screen as colored circles with diameters of $2 \mathrm{~cm}$
(Fig. 2-right). Subjects were asked to perform the goal-directed planar reaching movements with one continuous stroke from the central target to the peripheral targets and back, starting at the "North" position and proceeding clockwise. The movement was divided into sixteen reaching movements (8 directions, backward and forward movements) by processing off-line the manipulandum speed. Specifically, the movement was considered to start as the velocity first exceeded a threshold of $2 \%$ of peak velocity; conversely, the movement ended when the velocity dropped below the same threshold and the pointer was already inside the target [42]. The task was repeated five times.

At the beginning of the experimental protocol the subjects familiarized themselves with the robot and the required movements, performing three $\mathrm{CW}$ and three CCW circle drawing tasks, and three point-topoint tasks. These movements were not analyzed in this paper.

In order to evaluate the kinematics of the upper limb, a biomechanical model was developed. It consisted of three segments - trunk, upper arm, and forearm - connected together through the shoulder and elbow joints. The shoulder was modeled as a ball and socket joint with 3 DOFs while the elbow was modeled as a hinge joint. We assumed that centers of rotation of both joints were fixed and we ignored the forearm prono-supination and all the DOFs of the wrist due to the use of the orthotic device previously mentioned. Shoulder angles were computed following the approach described in Krabben et al. [23], based on the ISB recommendations [55]; specifically, we computed: 
the orientation of the plane of elevation $\left(\theta_{\operatorname{sh} 1}\right)$; the elevation and the axial rotation of the shoulder joint $\left(\theta_{\mathbf{s h} 2}\right)$ and, finally, the flex-extension angle of the elbow joint $\left(\theta_{\mathbf{e l}}\right)$. It is worth noting that preliminary tests were conducted in order to check for differences between groups in mean ROMs. Results showed that, during the circle trials, the mean ROM for all the computed angles produced similar results for adults and children (adults: $\theta_{\mathbf{s h} 1}=31 \pm 7^{\circ} ; \theta_{\mathbf{s h} 2}=14 \pm 5^{\circ} ; \theta_{\mathrm{el}}=$ $32^{\circ} \pm 7^{\circ}$; children: $\theta_{\text {sh1 }}=34^{\circ} \pm 6^{\circ} ; \theta_{\text {sh2 }}=17 \pm 7^{\circ}$; $\theta_{\text {el }}=37^{\circ} \pm 12^{\circ}$ ). Also the $\mathrm{P} 2 \mathrm{P}$ preliminary test showed the absence of significant differences between groups for $\theta_{\mathbf{s h} 1}, \theta_{\mathbf{s h} 2}$ and $\theta_{\mathrm{el}}$ (adults: $\theta_{\mathbf{s h} 1}=$ $17^{\circ} \pm 9^{\circ} ; \theta_{\mathbf{s h} 2}=6 \pm 4^{\circ} ; \theta_{\mathbf{e l}}=17^{\circ} \pm 10^{\circ} ;$ children: $\theta_{\mathbf{s h} 1}=20^{\circ} \pm 10^{\circ} ; \theta_{\mathbf{s h} 2}=8 \pm 5^{\circ} ; \theta_{\mathbf{e l}}=22^{\circ} \pm 12^{\circ}$ ).

Torques acting at the shoulder and the elbow were computed by using the above-described biomechanical model, the force measurements, and a Newton-Euler recursive algorithm. The required anthropometric measures - i.e. center of mass, weight and moment of inertia for each body segment - were deduced from literature data [54].

\subsection{Motor performance indices}

Studies of sensory-motor performance have identified a multitude of indices to quantify accuracy, smoothness and motor coordination for investigating the effect of age, disease, or therapeutic intervention. In addition, the gathered recordings from the robotic system and the optical tracking system represent a large amount of raw biomechanical data that should be processed to capture relevant distinctive features between children and adults subjects. Thus, the full analysis of the upper limb conducted here permitted the computation of a set of kinematic and kinetic variables evaluated in previous studies only on data provided by the robot and with simplifying assumptions on the upper limb kinematics [5, 7, 9, 18, 42, 47].

\subsubsection{Kinematic indices}

As regards the circle-drawing task, to characterize movement accuracy, smoothness, and synergy between shoulder and elbow joints, we have selected three indices. The Point Into Area (PIA) [18] expresses the percentage of points of the trajectory inside an annular region around the desired trajectory. As regards the selected threshold value, we tested the value of $1 \mathrm{~cm}$ proposed by Frascarelli et al. [18] and we lowered it to $0.5 \mathrm{~cm}$ and $0.1 \mathrm{~cm}$. However, considering the overall RMS error associated to the optoelectronic system $(\sim 1 \mathrm{~mm})$, we did not go below this threshold. The Speed Metric (SM) [42] is defined as the ratio between the mean speed and the maximum speed. Finally, the Joint Angles Correlation Metric (JACM) [7] is defined as:

$$
\mathrm{JACM}=\frac{\mathbf{C}\left(\theta_{\mathbf{1}}, \theta_{\mathbf{2}}\right)}{\sqrt{\mathbf{C}\left(\theta_{\mathbf{1}}, \theta_{\mathbf{1}}\right) \cdot \mathbf{C}\left(\theta_{\mathbf{2}}, \theta_{\mathbf{2}}\right)}}
$$

where $\mathbf{C}$ is the covariance matrix, $\theta_{\mathbf{1}}$ is the angle relating to the orientation of the plane of elevation of the shoulder, and $\theta_{\mathbf{2}}$ is the flex-extension angle of the elbow.

With respect to the point-to-point task, to characterize movement accuracy and smoothness, we have selected the following indices: the Position Error (PE) [5] is determined by the ratio of the actual length path and the theoretical one, which is the distance from the starting to the ending target; the Normalized Jerk (NJ) [47] is expressed by the following equation:

$$
\mathrm{NJ}=\sqrt{\frac{1}{2} \int \mathbf{j}^{2} \cdot \frac{T^{5}}{\left(\sum d R\right)^{2}} \cdot d t}
$$

where $\mathbf{j}$ is the jerk and $T$ is the duration of the movement.

Thus, movement accuracy was measured by means of PIA and PE (lower values of PIA and higher values of PE correspond to trajectories affected by lower accuracy); movement smoothness was quantified by means of SM and NJ (higher values of SM and lower values of NJ indicate smoother movements); synergy between shoulder and elbow joints was evaluated using JACM (lower values indicate higher independence between joints).

\subsubsection{Kinetic index}

Kinetic data, for both tasks, were analyzed by means of the Torque Sign (TS) index, proposed by Dounskaia et al. [9]. In order to evaluate TS for shoulder and elbow joints, the torque estimated at each joint was articulated in the following components: net torque (NT), proportional to angular acceleration at the joint; interactive torque (IT), depending on motion at both joints; and, finally, generalized muscle torque (GMT), which includes the muscle activity and the viscoelastic properties of the entire arm. Gravitational torque was not taken into account, since movement was restrained to the horizontal plane. The relationship between the previously cited torque components is, for the elbow: 


$$
\mathrm{GMT}_{\mathrm{e}}=\mathrm{NT}_{\mathrm{e}}-\mathrm{IT}_{\mathrm{e}}
$$

while for the shoulder:

$$
\mathrm{GMT}_{\mathrm{s}}=\mathrm{NT}_{\mathrm{s}}-\mathrm{IT}_{\mathrm{s}}-\mathrm{GMT}_{\mathrm{e}}
$$

In the $\mathrm{IT}_{\mathrm{e}}$ term, we included also the interaction between the subjects and the end-effector, taking into account forces measured by the six-component load cell of the robot.

Finally, TS was computed as the percentage of time when GMT had the same sign of NT and it represents the percentage of time in which each joint gives a positive contribution to movement acceleration. Higher values of the index TS represent a higher contribution of the muscles to produce the net joint displacement.

\subsection{Statistical analysis}

All data were tested for normality with the ShapiroWilk test. The significance level was set at 0.05 for all statistical tests. All the kinematic indices chosen for the circle-drawing task were analyzed with GROUP $\times$ ROTATION, i.e. $2 \times 2$, mixed ANOVA design tests. We used GROUP (adults vs. children) as between-subjects factor and ROTATION (CW vs. $\mathrm{CCW}$ ) as within-subjects factor to investigate whether the rotation direction affects the motor control of children and adults in a different way.

As regards the point-to-point task, a GROUP $\times$ DIRECTION, i.e. $2 \times 8$ mixed ANOVA tests, were performed on the kinematic indices. Specifically, we used GROUP (adults vs. children) as a between-subjects factor and DIRECTION (N, NE, E, SE, S, SW, W, and NW) as a within-subjects factor to investigate whether the directional distribution of the kinematic variables is similar in the two groups. We adopted the Greenhouse-Geisser correction when the Mauchly's test was significant and the assumption of sphericity was violated. If the interaction effect GROUP $\times$ DIRECTION was significant, we broke down the interactions comparing children and adults with paired $t$-tests at each level of DIRECTION.

Torque sign (TS) was analyzed with GROUP $\times$ JOINT, i.e. $2 \times 2$, mixed ANOVA design tests. We used GROUP (adults vs. children) as between-subjects factor and JOINT (elbow vs. shoulder) as within-subjects factor. The ANOVA tests were conducted separately for each rotation (circle-drawing task) and direction (point-to-point task).
Statistical analyses were performed with built-in functions of SPSS 20 (IBM, Armonk, NY).

\section{Results}

\subsection{Circle-drawing task}

The upper panels of Fig. 3 show the end-effector trajectories of representative trials collected with a child and an adult during the execution of the circle-drawing task. Kinematic indices are reported in Fig. 4. Regarding the movement accuracy index Point Into Area PIA, the GROUP $\times$ ROTATION mixed ANOVA test showed the main effect GROUP and the interaction factor were not statistically different for the three selected threshold values $(1 \mathrm{~cm}$, $0.5 \mathrm{~cm}$ and $0.1 \mathrm{~cm})$. Therefore, we reported results only for the $0.1 \mathrm{~cm}$ threshold value since it represents the most challenging condition for both groups. Moreover, children and adults showed a higher value for PIA $(p<0.001)$ in the CW condition (children: $80.4 \pm 12.2 \%$; adults: $75.9 \pm 13.9 \%$ ) than in the $\mathrm{CCW}$ one (children: $66.5 \pm 19.4 \%$; adults: $66.6 \pm 17.9 \%$ ). The smoothness index Speed Metric SM was higher for adults than children $(p<0.001)$, for both $\mathrm{CW}$ (children: $0.43 \pm 0.06$; adults: $0.59 \pm 0.08)$ and CCW direction (children: $0.41 \pm 0.07$; adults: $0.59 \pm 0.08$ ), while the main effect ROTATION $(p=0.562)$ and the interaction factor $(p=0.430)$ were not significant. The coordination index Joint Angle Correlation Metric JACM did not show significant main effects (GROUP: $p=0.385$, ROTATION: $p=0.591$ ) nor did the interaction factor $(p=0.463)$.

Figure 5 shows the results for the kinetic index Torque Sign TS at shoulder and elbow. TS at shoulder is always higher than at elbow $(p<0.001)$ for both CW (shoulder: children, $92.47 \pm 3.16 \%$; adults, $92.28 \pm 3.21 \%$; elbow: children, $48.41 \pm 5.21 \%$; adults, $49.41 \pm 6.20)$ and $\mathrm{CCW}$ directions (shoulder: children, $92.17 \pm 2.99 \%$; adults, $89.76 \pm 4.94 \%$; elbow: children, $49.01 \pm 3.84 \%$; adults, $49.90 \pm 7.22 \%$ ). The interaction factors were all not significant (CW: $p=0.478$; CCW: $p=0.316$ ) and no differences between groups were found $(\mathrm{CW}$ : $p=0.614$; CCW: $p=0.353$ ).

\subsection{Point-to-point task}

The lower panels of Fig. 3 show the end-effector trajectories of representative trials collected with a 

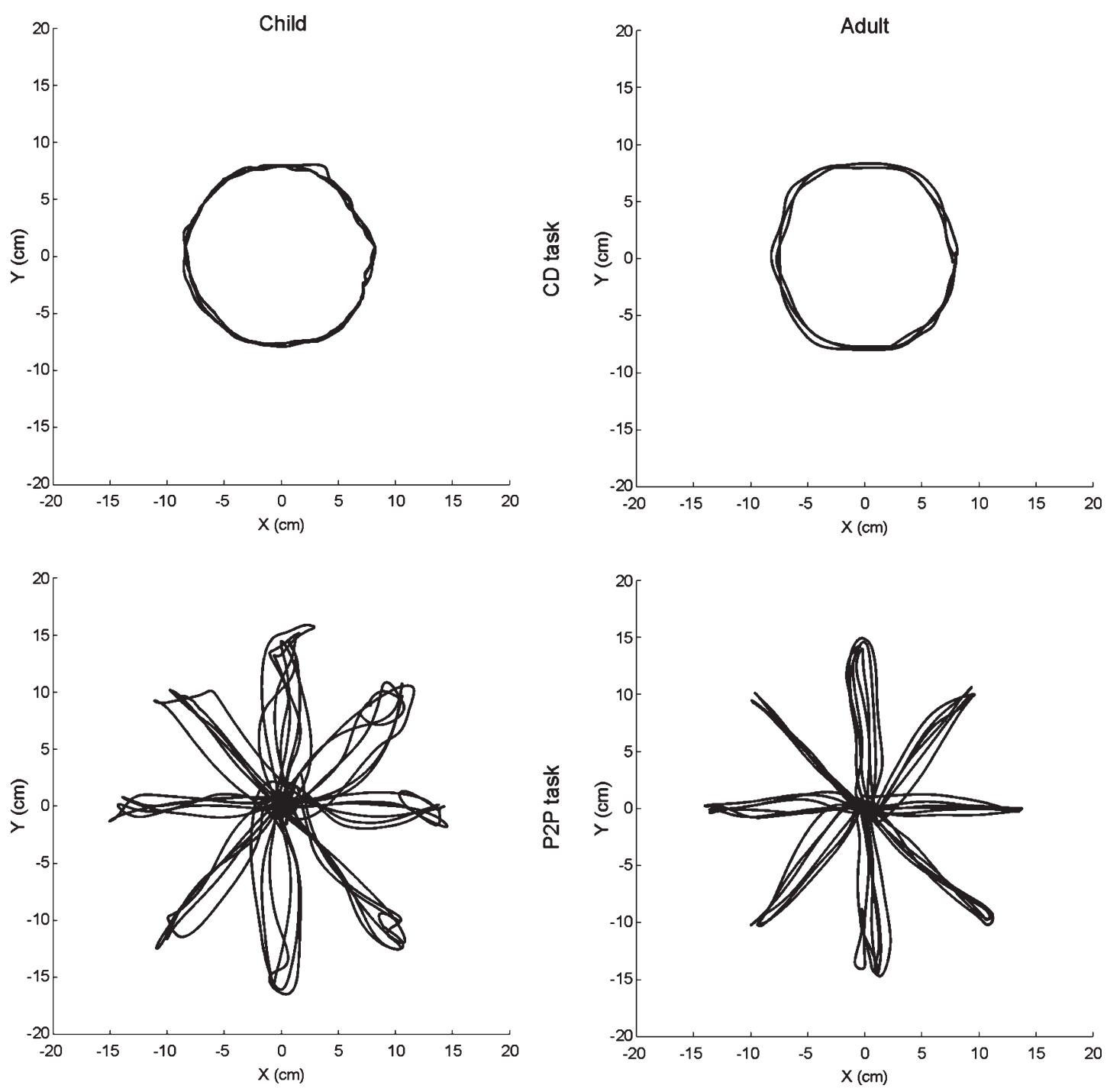

Fig. 3. Paths traced by two representative subjects from groups of children and adult: (upper panels) circle-drawing task in clockwise and counter-clockwise directions, (lower panels) point-to-point task.

child and an adult during the execution of a pointto-point task. The Position Error PE and Normalized Jerk NJ are reported in Fig. 6. An ANOVA mixed test performed on the index PE showed significant differences between groups $(p<0.001)$, with higher values for children (children, E: $1.08 \pm 0.05 ; \mathrm{N}: 1.17 \pm 0.10$; NE: $1.12 \pm 0.06 ;$ SW: $1.09 \pm 0.09 ;$ SE: $1.10 \pm 0.12 ;$ S: $1.09 \pm 0.07 ; \mathrm{W}: 1.06 \pm 0.04 ; \mathrm{NW}: 1.10 \pm 0.11 ;$ adults, $\mathrm{E}: 1.04 \pm 0.03 ; \mathrm{N}: 1.02 \pm 0.01 ; \mathrm{NE}: 1.06 \pm 0.04$; SW: $1.03 \pm 0.02$; SE: $1.04 \pm 0.04 ; \mathrm{S}: 1.03 \pm 0.02$; W: $1.04 \pm 0.03$; NW: $1.06 \pm 0.16)$. Conversely, DIRECTION $(p=0.310)$ and the interaction factor $(p=0.158)$ were not statistically meaningful. As regards $\mathrm{NJ}$, GROUP $(p<0.01)$ and DIRECTION $(p<0.05)$ together with the interaction factor $(p<0.01)$ showed a statistically significant difference. More specifically, children showed higher values of $\mathrm{NJ}$ than adults (children, E: $69.39 \pm 33.81 ; \quad \mathrm{N}: 62.92 \pm 25.68$; NE: $\quad 54.85 \pm 36.87 ; \quad$ SW: $45.46 \pm 24.36 ; \quad$ SE: $59.36 \pm 43.98 ;$ S: $81.67 \pm 43.22 ; \mathrm{W}: 43.65 \pm 28.59$; NW: $91.53 \pm 52.53$; adults, E: $25.22 \pm 18.00 ; \mathrm{N}$ : $37.22 \pm 26.07$; NE: $26.98 \pm 15.79$; SW: $20.56 \pm 8.51$; SE: $26.02 \pm 9.84$; S: $33.86 \pm 22.31$; W: $26.53 \pm 12.09$; NW: $18.83 \pm 6.63$ ), while $T$-tests revealed significant 

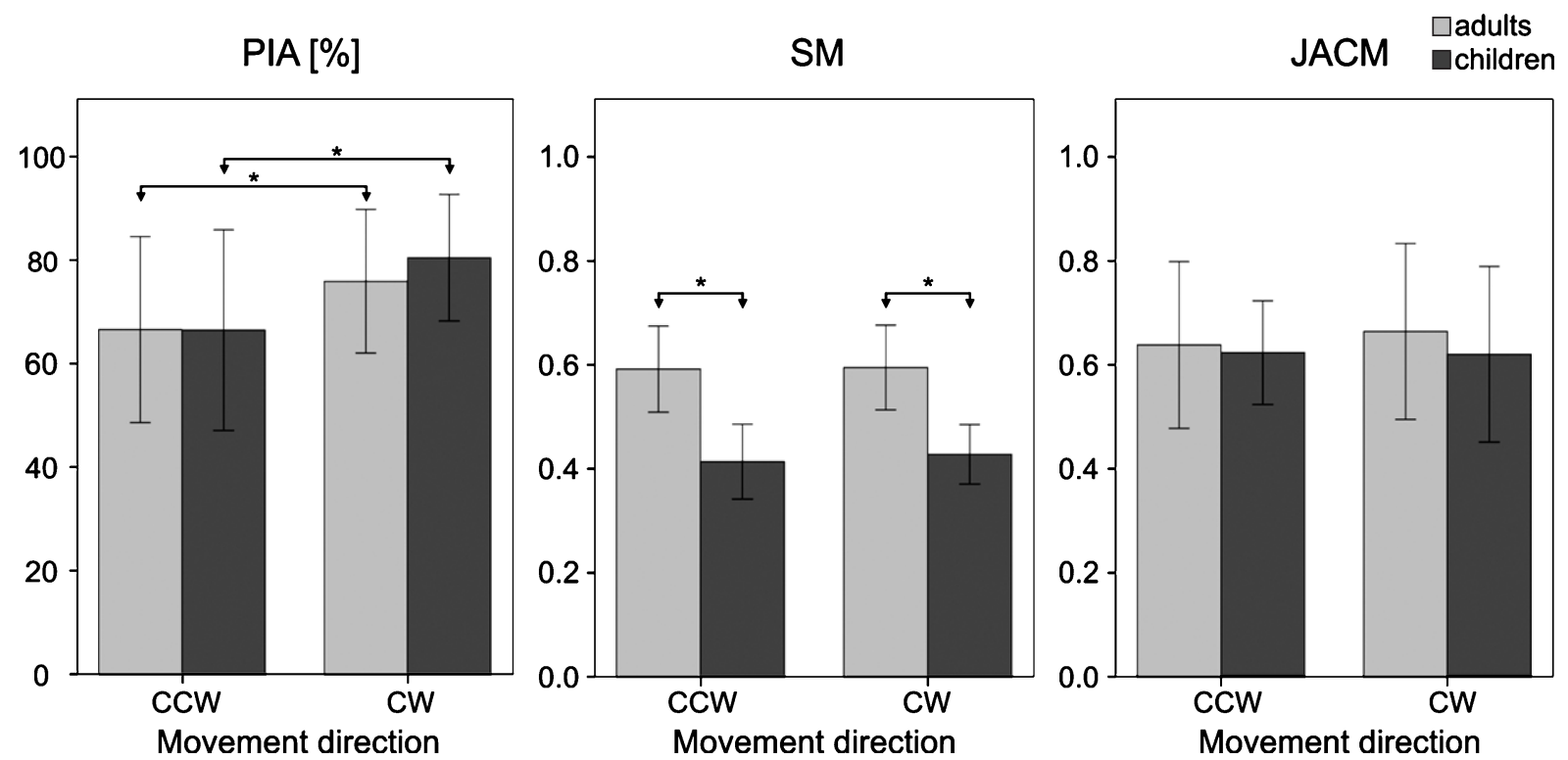

Fig. 4. Mean and standard deviation for Point in Area (PIA), Speed Metric (SM), and Joint Angles Correlation Metric (JACM) indices, for each group and direction. The symbol ${ }^{*}$ indicates when two values are significantly different.

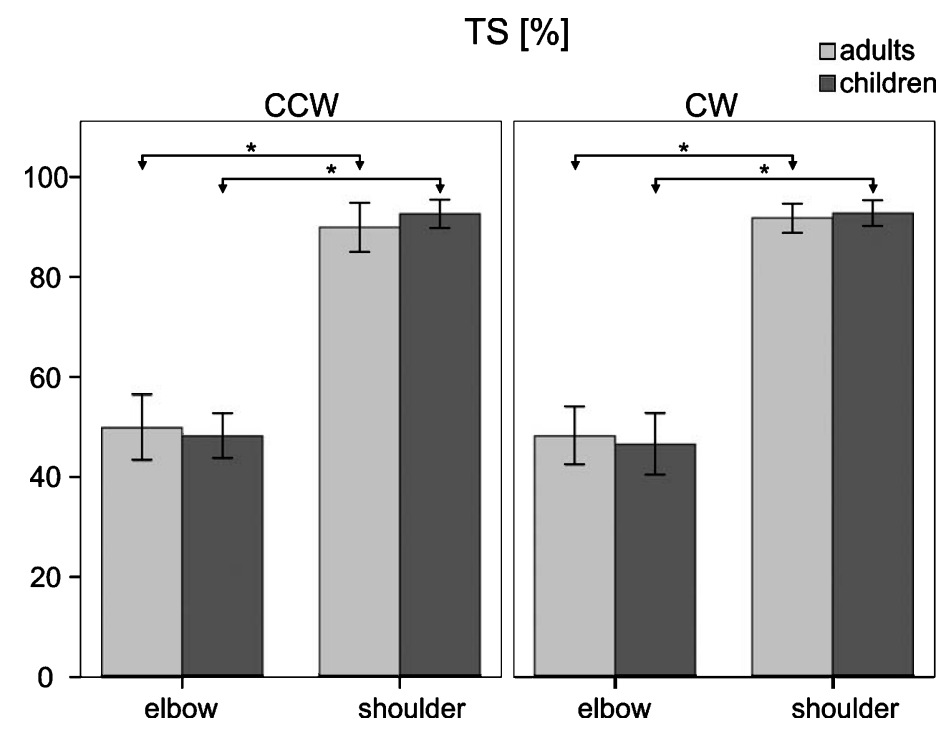

Fig. 5. Mean and standard deviation for Torque Sign (TS) index, for each group, joint, and direction. The symbol * indicates when elbow is significantly different from shoulder.

differences between adults and children for each direction (E: $p<0.001 ; \mathrm{N}: p<0.05 ; \mathrm{NE}: p<0.05$; SW: $p<0.01 ;$ SE: $p<0.05 ;$ S: $p<0.01 ; \quad$ NW: $p<0.001)$, with the exception of the $\mathrm{W}$ condition $(p=0.07)$.

Kinetic index torque sign TS for the shoulder and elbow is reported in Fig. 7. For each direction, TS at the shoulder is higher than at the elbow $(p<0.001)$ for both groups and no differences between groups were found $(\mathrm{N}: p=0.262$; NE: $p=0.320 ; \mathrm{E}: p=0.852 ; \mathrm{SE}: p=0.754 ; \mathrm{S}: p=0.517$; SW: $p=0.321$; $: p=0.836$; NW: $p=0.122$; the mean values across direction are, for children $48.83 \pm 13.17$ and for adults $48.69 \pm 15.43$ ). Also the interaction factors were not significant for each direction $(\mathrm{N}$ : $p=0.452$; NE: $p=0.190 ; \mathrm{E}: p=0.798$; SE: $p=0.280$; 
PE

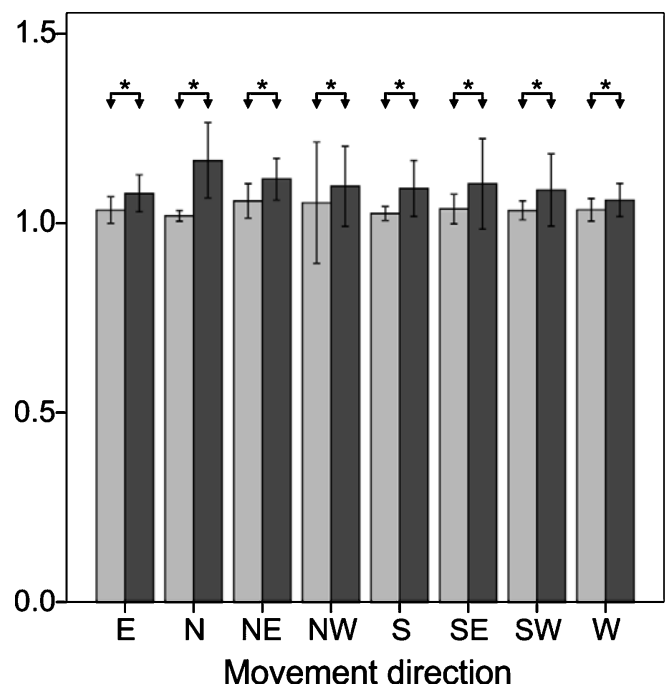

NJ

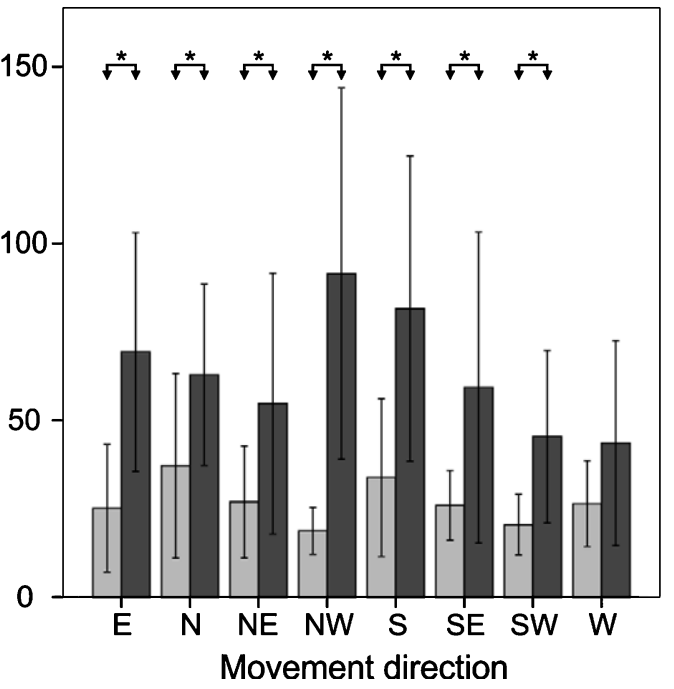

Fig. 6. Mean and standard deviation for Position Error (PE) and Normalized Jerk (NJ) indices, for each group and direction. The symbol * indicates when the children are significantly different from adults.

S: $p=0.645 ; \mathrm{SW}: p=0.444 ; \mathrm{W}: p=0.730 ; \mathrm{NW}$ : $p=0.927$ ).

\section{Discussion}

In this study, we quantified the differences between normally developed children and young healthy subjects in the upper limb movements while performing tracking tasks and multi-direction reaching movements with a backdriveable low-friction rehabilitation robot. Thus, the machine did not interfere with motion and allowed the individual to move the manipulandum freely.

The main finding of this study was that children aged 7 to 10 years have the same joint coordination and torque control as adults, but they developed an adult-like accuracy only in tracking tasks. Moreover, the distinguishing feature of upper arm movements in children is the lack of smoothness; in fact, it was always lower than that of adults for both motor tasks.

In the following subsections the movement coordination, accuracy and smoothness are separately discussed.

\subsection{Movement coordination}

The quantification of the shoulder and elbow torques allows us to interpret the control strategy used by the
CNS to move the limb. In particular, the analysis of Torque Sign TS aimed to reveal whether there are differences between adults and children in the role of torque control for the production of motion at the elbow and shoulder joints. The index TS assumed higher values at the shoulder joint for both groups, and for both motor tasks independently of the movement direction. Therefore, the shoulder can be considered the leading joint, while the elbow is the subordinate joint. Hence, at the elbow level the role of Generalized Muscle Torque GMT is to adjust the motion at this joint, correcting the Interactive Torque IT in order to efficiently complete the motor task.

Moreover, the absence of significant differences between the two groups might suggest that in normally developed children aged 7 to 10 years the two major stages of motor learning - i.e. the exploitation of the biomechanical properties of the leading and the subordinate joint - are already achieved.

The maturity of children's motor strategy is also suggested by an exam of Joint Angles Correlation Metric JACM values in the circle drawing task, which are not significantly different from those of adults.

These findings suggest that children's CNS correctly blends both proprioceptive and visual information, which has already been shown to be crucial in upper limb joint coupling and coordination [43]. 

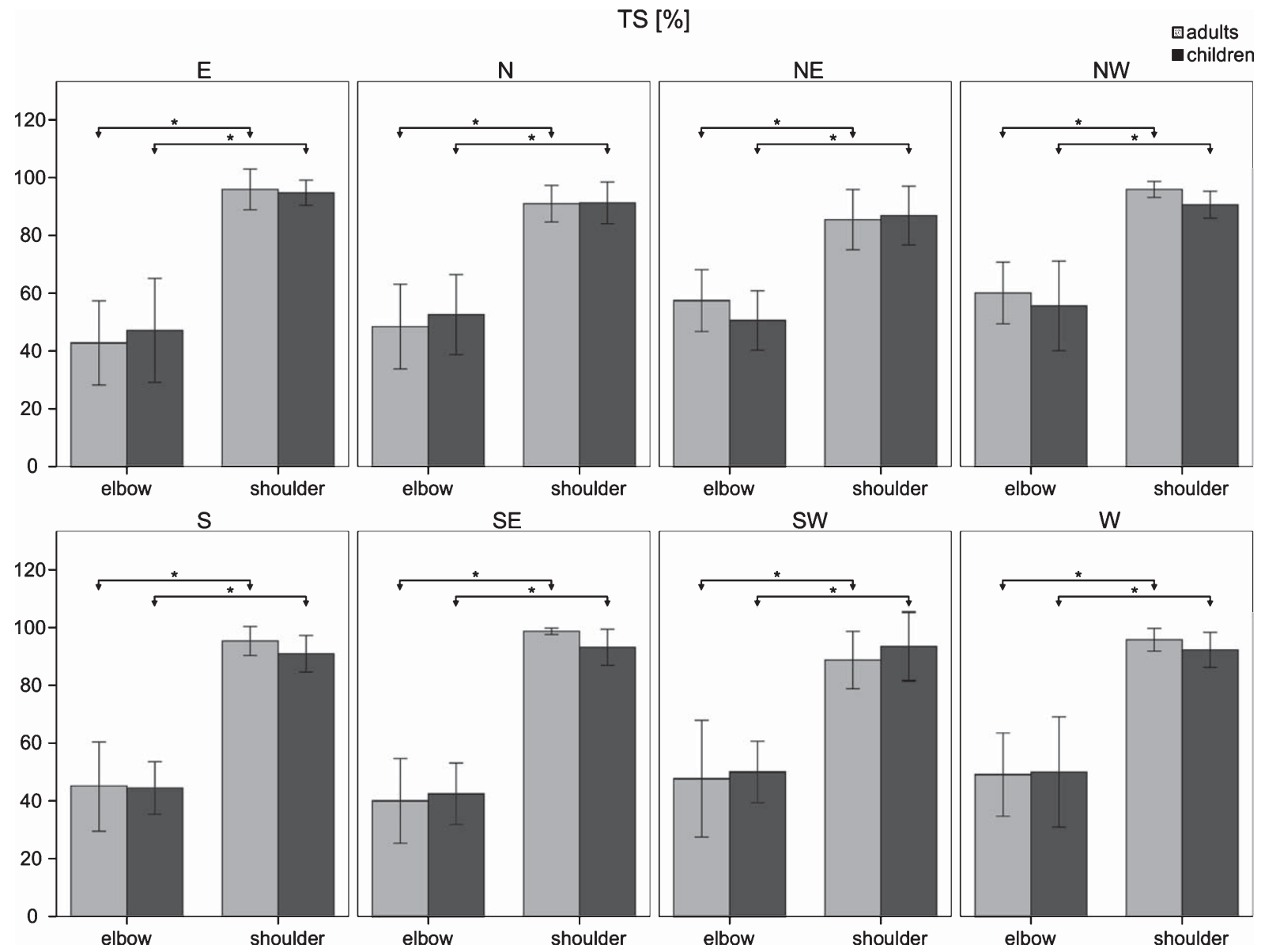

Fig. 7. Mean and standard deviation for Torque Sign (TS) index, for each group, joint, and direction. The symbol * indicates when elbow is significantly different from shoulder.

\subsection{Movement accuracy}

As regards the drawing circle tasks, the lack of significant differences between groups in the index Point Into Area PIA suggests that the visuomotor system of children is capable of using, in an adult-like manner, visual information to evaluate the disparity between the performed and the desired trajectories reported on the monitor, and to correct the ongoing movement. This outcome confirms the findings of Robertson [40] in which the movement accuracy is not different between 8-year-olds and adults during circle drawing. Indeed, children reveal the same tendency as adults, tracing more accurate circles in the clockwise direction than in counterclockwise one.

Conversely, during reaching tasks, the significant differences between the two groups in the Position
Error PE clearly shows that an adult-like accuracy is not yet achieved by children aged 7 to 10 years. Moreover, no differences emerged among the directions and no significant interactions were found: this can be interpreted as children need to improve their capacity for making straight paths through the entire workspace here examined. Our findings confirm the decrease in accuracy in children during reaching movement, as reported by Thomas et al. [48]. Interestingly, our results stand in contrast to the results of Schneiberg et al. [44], in which children aged 6 to 8 years attained adult-level accuracy. It can be explained by the different kinds of required reaching tasks which, in the Schneiberg's study, were performed in a sagittal plane. It confirms that the complete development of motor control is achieved at different ages as a function of the plane where the movement is performed. 


\subsection{Movement smoothness}

As regards the circle-drawing task, the Speed Metric SM index was significantly lower in children than in adults in both directions: this result demonstrates that children perform the required movement assigning higher priority to accuracy with respect to smoothness.

During reaching movements, the Normalized Jerk $\mathrm{NJ}$ index was lower in children than in adults. This result demonstrates that movement smoothness is not yet maximized by children aged 7 to 10 years irrespective of the required motor task. The present finding is consistent with the results of Traynor et al. [49] in which smoothness decreases with developmental age and assumes the lowest value in adults. Moreover, smoothness in point-to-point task varies among the reaching directions. Specifically, in the $\mathrm{W}$ direction, the Normalized Jerk NJ is not statistically different between groups and it assumes the lowest value for the children. The effect of different directions on movement smoothness confirms the outcomes of other studies $[6,14,19,31]$ that highlighted the relationships between the inertial anisotropy of the upper arm and the spatial distribution of the kinematic pattern. Specifically, they found that, in the direction characterized by higher values of arm inertia, movements are performed with higher velocity, higher acceleration and less duration; instead, in the direction perpendicular to the previous one, the kinematic error is lower. In our case, the lower smoothness showed by children could be because it is harder for them to adequately compensate for changes in limb inertia related to the change in movement direction, and this is especially evident in the direction with lower values of inertia. In fact, in the W direction, where inertia is higher [19], there are no differences between the two groups and therefore we could infer that here the upper limb configuration is the easiest for children to manage. As regards the influence of movement direction on motor performance, it is worth noting that a similar behavior was also found at the ankle, where the movements in the dorsi/plantar flexion direction were performed with different kinematic patterns compared with those performed in inversion/eversion direction [34]; in that case, however, the differences were related both to a different control capability in the two analyzed directions, and to the mechanical properties of the joint, rather than to different inertia of the joint.

Children in the selected age range show greater movement variability in all directions, as assessed by the mean standard deviation of the NJ. This is consistent with the results obtained by Takahashi et al. [46] who considers the motor performance limitations in children caused by an incomplete definition of the planning process due to the body changes of developmental age.

From a global examination of the collected data the children aged 7 to 10 years perform arm movements with a lower smoothness than adults. How could we explain it? A widely shared idea affirms that the CNS plans the motor strategy minimizing critical parameters of trajectory (e.g. jerk [15], or torque [50]) in order to achieve an accurate and smooth movement. This hypothesis is supported by the smoothness decrease in patients with neurological disease such as stroke survivors [42] or children with cerebral palsy [25] compared with healthy subjects. Following that hypothesis, our results could be ascribed to an incomplete definition of the movement planning performed by the CNS, due to a developing nervous system [46]. However, the main role of the CNS in generating smooth trajectories has been questioned by other authors [20, 26] who have suggested that the intrinsic properties of active muscle tissue may be sufficient to produce smooth motion, even in the absence of specifically programmed neural inputs. Following that approach, our results could be related to the actual morphological muscular differences between adults and children, like force-velocity relationship, stiffness [52] and mass.

Our results cannot completely answer the previous question and, therefore, future study will seek to identify the roles played by the muscles and CNS to generate a smooth motor trajectory. Nevertheless, independently of the physiological mechanisms underlying the observed difference, our data extend the previous results on elderly subjects that showed a degradation of smoothness with ageing $[5,56]$, pointing out the importance of evaluating the motor performance of pediatric patients with an age-matched control group.

Therefore, from a rehabilitation perspective, our findings lead to new insights in motor rehabilitation. Firstly, as regards children, the quantitative performance indices used to assess changes due to the pathological impairment could lead to a more specific, patient-tailored rehabilitation plan. Moreover, on the basis of our results, one might speculate that the degree of assistance that the robot provides during the therapy session should be varied according to the developmental stage of subjects' motor control and the required 
motor task [33], in order to enhance beneficial therapeutics effects. Secondly, our results may lead to an improvement of the controllers for the rehabilitation robots, especially for subjects with stroke. Previous works on the design of control algorithms for rehabilitation robots were based on the hypothesis that the recovery process is similar to the motor learning process [33]. The last one was found to be crucial during the recovery and better characterizes, compared to an adaptation process, the improvements of the patients with stroke during the training with the robotic devices [8]. Therefore, the comprehension, in term of kinematics and kinetics, of the step by step process that brings children to an adult-like motor behavior, could be useful to design controllers that make the therapy more effective by developing more natural rehabilitation scenarios.

One must refer to our findings with the appropriate caveats. When comparing pathological children with a control group there is a greater variability of performance indicators due to both the pathology and the motor control development which usually proceeds at different times. One could take into account for this problem by increasing the number of the subjects involved in the control group and/or grouping children in different age ranges. The well choice of the age range is a crucial issue in studies which compare children with pathology and healthy subjects. In fact if the age range is large, the variability of the control group increases and it could hide a difference related to the pathology. Conversely, if the age range is small it is possible to misinterpret a difference related to motor control development with a difference induced by pathology.

\section{Conclusions}

This study aimed to compare the motor skills of normally developed children and healthy adults performing two tasks usually employed in robot-mediated therapy. Our results, which take advantage of the integration of the robotic device and the optical tracking system, show that children aged 7 to 10 years have the same level of motor coordination of adults and prefer to optimize the accuracy with respect to smoothness during the circle-drawing task. Moreover, they perform both tasks with a less smooth trajectory.

In conclusion, the findings of this study provide evidence for the current practice of quantifying the recovery of arm motion during rehabilitation with agematched subjects among pediatric patients.

\section{Acknowledgments}

This study formed part of the $\mathrm{PhD}$ program pursued by AP at University of Padua (Italy) and by MG at the "Sapienza" University of Rome (Italy). This research is supported by a grant from the Italian Ministry of Public Education, University and Research MIUR ("Robotic systems for the rehabilitation" - year 2003/07; Principal Investigator: PC) and a grant from the Italian Ministry of Health ("Pilot study on a new class of medical devices: robotic system for rehabilitation and rehabilitation" - year 2009/11; Principal Investigator: PC). We wish to thank all the participants who agreed to voluntarily participate in the experiment.

\section{References}

[1] B.R. Brewer, R. Klatzky and Y. Matsuoka, 'Initial Therapeutic Results of Visual Feedback Manipulation in Robotic Rehabilitation', in, Virtual Rehabilitation, 2006 International Workshop on, (2006).

[2] B.R. Brewer, S.K. McDowell and L.C. Worthen-Chaudhari, Poststroke upper extremity rehabilitation: A review of robotic systems and clinical results, Topics in stroke rehabilitation 6(14) (2007), 22-44.

[3] M. Casadio, P. Morasso, A.N. Ide, V. Sanguineti and P. Giannoni, Measuring functional recovery of hemiparetic subjects during gentle robot therapy, Measurement 8(42) (2009), 11761187.

[4] E. Castelli, F. Frascarelli, L. Masia, G. Di Rosa, M. Petrarca and P. Cappa, Robotic rehabilitation of upper limbs in children, Journal of Head Trauma Rehabilitation 5(23) (2008), 341-341.

[5] B. Cesqui, G. Macri, P. Dario and S. Micera, Characterization of age-related modifications of upper limb motor control strategies in a new dynamic environment, Journal of Neuroengineering and Rehabilitation 5 (2008), 31-44.

[6] M. Darainy, A. Mattar and D.J. Ostry, Effects of human arm impedance on dynamics learning and generalization, J Neurophysiol,6(101) (2009), 3158.

[7] L. Dipietro, H.I. Krebs, S.E. Fasoli, B.T. Volpe, J. Stein, C. Bever and N. Hogan, Changing motor synergies in chronic stroke, Journal of Neurophysiology 2(98) (2007), 757-768.

[8] L. Dipietro, H. Krebs, B. Volpe, J. Stein, C. Bever, S. Mernoff, S. Fasoli and N. Hogan, Learning, not adaptation, characterizes stroke motor recovery: Evidence from kinematic changes induced by robot-assisted therapy in trained and untrained task in the same workspace, Neural Systems and Rehabilitation Engineering, IEEE Transactions on 1(20) (2012), 48-57.

[9] N. Dounskaia, C.J. Ketcham and G.E. Stelmach, Commonalities and differences in control of various drawing movements, Exp Brain Res 1(146) (2002), 11-25. 
[10] S.E. Fasoli, H.I. Krebs, J. Stein, W.R. Frontera and N. Hogan, Effects of robotic therapy on motor impairment and recovery in chronic stroke, Archives of Physical Medicine and Rehabilitation 4(84) (2003), 477-482.

[11] S.E. Fasoli, H.I. Krebs, J. Stein, W.R. Frontera, R. Hughes and N. Hogan, Robotic therapy for chronic motor impairments after stroke: Follow-up results, Archives of Physical Medicine and Rehabilitation 7(85) (2004), 1106-1111.

[12] S.E. Fasoli, M. Fragala-Pinkham, R. Hughes, N. Hogan, H.I. Krebs and J. Stein, Upper Limb Robotic Therapy for Children with Hemiplegia, American Journal of Physical Medicine and Rehabilitation 11(87) (2008), 929-936.

[13] M. Ferraro, J.J. Palazzolo, J. Krol, H.I. Krebs, N. Hogan and B.T. Volpe, Robot-aided sensorimotor arm training improves outcome in patients with chronic stroke, Neurology 11(61) (2003), 1604-1607.

[14] J.R. Flanagan and S. Lolley, The inertial anisotropy of the arm is accurately predicted during movement planning, The Journal of Neuroscience 4(21) (2001), 1361-1369.

[15] T. Flash and N. Hogan, The coordination of arm movements an experimentally confirmed mathematical-model, Journal of Neuroscience 7(5) (1985), 1688-1703.

[16] G.G. Fluet, Q. Qiu, D. Kelly, H.D. Parikh, D. Ramirez, S. Saleh and S.V. Adamovich, Interfacing a haptic robotic system with complex virtual environments to treat impaired upper extremity motor function in children with cerebral palsy, Dev Neurorehabil 5(13) (2010), 335-345.

[17] F. Frascarelli, L. Masia, G. Di Rosa, P. Cappa, M. Petrarca, E. Castelli and H.I. Krebs, The impact of robotic rehabilitation in children with acquired or congenital movement disorders, European Journal of Physical and Rehabilitation Medicine 1(45) (2009), 135-141.

[18] F. Frascarelli, L. Masia, G. Di Rosa, M. Petrarca, P. Cappa and E. Castelli, Robot-mediated and clinical scales evaluation after upper limb botulinum toxin type a injection in children with hemiplegia, Journal of Rehabilitation Medicine 12(41) (2009), 988-994.

[19] J. Gordon, M.F. Ghilardi, S.E. Cooper and C. Ghez, Accuracy of planar reaching movements, Experimental Brain Research 1(99) (1994), 112-130.

[20] P.L. Gribble and D.J. Ostry, Origins of the power law relation between movement velocity and curvature: Modeling the effects of muscle mechanics and limb dynamics, Journal of Neurophysiology 5(76) (1996), 2853-2860.

[21] P. Jansen-Osmann, S. Richter, J. Konczak and K.T. Kalveram, Force adaptation transfers to untrained workspace regions in children - Evidence for developing inverse dynamic motor models, Experimental Brain Research 2(143) (2002), 212-220.

[22] J. Konczak, P. Jansen-Osmann and K.T. Kalveram, Development of force adaptation during childhood, Journal of motor behavior 1(35) (2003), 41-52.

[23] T. Krabben, B.I. Molier, A. Houwink, J.S. Rietman, J.H. Buurke and G.B. Prange, Circle drawing as evaluative movement task in stroke rehabilitation: An explorative study, Journal of Neuroengineering and Rehabilitation 8 (2011), 15.

[24] H.I. Krebs, B. Ladenheim, C. Hippolyte, L. Monterroso and J. Mast, Robot-assisted task-specific training in cerebral palsy, Developmental Medicine \& Child Neurology s4(51) (2009), 140-145.

[25] H.I. Krebs, S.E. Fasoli, L. Dipietro, M. Fragala-Pinkham, R. Hughes, J. Stein and N. Hogan, Motor Learning Characterizes
Habilitation of Children With Hemiplegic Cerebral Palsy, Neurorehabilitation and Neural Repair 7(26) (2012), 855-860.

[26] A.M. Krylow and W.Z. Rymer, Role of intrinsic muscle properties in producing smooth movements, Ieee Transactions on Biomedical Engineering 2(44) (1997), 165-176.

[27] P.C. Kung, C.C.K. Lin and M.S. Ju, Neuro-rehabilitation robotassisted assessments of synergy patterns of forearm, elbow and shoulder joints in chronic stroke patients, Clinical Biomechanics 7(25) (2010), 647-654.

[28] A.C. Lo, P. Guarino, H.I. Krebs, B.T. Volpe, C.T. Bever, P.W. Duncan, R.J. Ringer, T.H. Wagner, L.G. Richards, D.M. Bravata, J.K. Haselkorn, G.F. Wittenberg, D.G. Federman, B.H Corn, A.D. Maffucci and P. Peduzzi, Multicenter Randomized Trial of Robot-Assisted Rehabilitation for Chronic Stroke: Methods and Entry Characteristics for VA ROBOTICS, $\mathrm{Neu}$ rorehabilitation and Neural Repair 8(23) (2009), 775-783.

[29] H.S. Lo and S.Q. Xie, Exoskeleton robots for upper-limb rehabilitation: State of the art and future prospects, Medical Engineering \& Physics 3(34) (2012), 261-268.

[30] R.C.V. Loureiro, W.S. Harwin, K. Nagai and M. Johnson, Advances in upper limb stroke rehabilitation: A technology push, Medical \& Biological Engineering \& Computing 10(49) (2011), 1103-1118.

[31] L. Masia, F. Frascarelli, P. Morasso, G. Di Rosa, M. Petrarca, E. Castelli and P. Cappa, Reduced short term adaptation to robot generated dynamic environment in children affected by Cerebral Palsy, Journal of Neuroengineering and Rehabilitation $\mathbf{8}$ (2011).

[32] J. Mehrholz, A. Hadrich, T. Platz, J. Kugler, and M. Pohl, Electromechanical and robot-assisted arm training for improving generic activities of daily living, arm function, and arm muscle strength after stroke, Cochrane Database of Systematic Reviews 6 (2012).

[33] K.P. Michmizos and H.I. Krebs, 'Assist-as-needed in lower extremity robotic therapy for children with Cerebral Palsy', in, Biomedical Robotics and Biomechatronics (BioRob), 2012 4th IEEE RAS \& EMBS International Conference on, (IEEE, 2012)

[34] K.P. Michmizos and H.I. Krebs, Pointing with the ankle: The speed-accuracy trade-off, Experimental Brain Research 2(232) (2014), 647-657.

[35] D. Novak, J. Ziherl, A. Olenšek, M. Milavec, J. Podobnik, M. Mihelj and M. Munih, Psychophysiological responses to robotic rehabilitation tasks in stroke, IEEE Transactions on Neural Systems and Rehabilitation Engineering 4(18) (2010), 351-361.

[36] I. Olivier, L. Hay, C. Bard and M. Fleury, Age-related differences in the reaching and grasping coordination in children: Unimanual and bimanual tasks, Experimental Brain Research 1(179) (2007), 17-27.

[37] J.L. Patton, M.E. Stoykov, M. Kovic and F.A. Mussa-Ivaldi, Evaluation of robotic training forces that either enhance or reduce error in chronic hemiparetic stroke survivors, Experimental Brain Research 3(168) (2006), 368-383.

[38] G.B. Prange, M.J.A. Jannink, C.G.M. Groothuis-Oudshoorn, H.J. Hermens and M.J. IJzerman, Systematic review of the effect of robot-aided therapy on recovery of the hemiparetic arm after stroke, Journal of rehabilitation research and development 2(43) (2006), 171-183.

[39] R. Riener, T. Nef and G. Colombo, Robot-aided neurorehabilitation of the upper extremities, Medical \& Biological Engineering \& Computing 1(43) (2005), 2-10. 
[40] S.D.R. Ringenbach and P.G. Amazeen, How do children control rate, amplitude, and coordination stability during bimanual circle drawing? Ecological Psychology 1(17) (2005), 1-18.

[41] S.D. Robertson, Development of bimanual skill: The search for stable patterns of coordination, J Mot Behav 2(33) (2001), 114-126.

[42] B. Rohrer, S. Fasoli, H.I. Krebs, R. Hughes, B. Volpe, W.R. Frontera, J. Stein and N. Hogan, Movement smoothness changes during stroke recovery, Journal of Neuroscience 18(22) (2002), 8297-8304.

[43] R.L. Sainburg, M.F. Ghilardi, H. Poizner and C. Ghez, Control of limb dynamics in normal subjects and patients without proprioception, Journal of Neurophysiology 2(73) (1995), 820835 .

[44] S. Schneiberg, H. Sveistrup, B. McFadyen, P. McKinley and M.F. Levin, The development of coordination for reach-tograsp movements in children, Experimental Brain Research 2(146) (2002), 142-154.

[45] N. Shirzad and H.F.M. Van der Loos, 'Error amplification to promote motor learning and motivation in therapy robotics', in, Engineering in Medicine and Biology Society (EMBC), 2012 Annual International Conference of the IEEE, (2012)

[46] C.D. Takahashi, D. Nemet, C.M. Rose-Gottron, J.K. Larson, D.M. Cooper and D.J. Reinkensmeyer, Neuromotor noise limits motor performance, but not motor adaptation, in children, Journal of Neurophysiology 2(90) (2003), 703-711.

[47] H.L. Teulings, J.L. ContrerasVidal, G.E. Stelmach and C.H Adler, Parkinsonism reduces coordination of fingers, wrist, and arm in fine motor control, Experimental Neurology 1(146) (1997), 159-170.

[48] J.R. Thomas, J.H. Yan and G.E. Stelmach, Movement substructures change as a function of practice in children and adults, Journal of Experimental Child Psychology 3(75) (2000), 228-244.
[49] R. Traynor, V. Galea and M.R. Pierrynowski, The development of rhythm regularity, neuromuscular strategies, and movement smoothness during repetitive reaching in typically developing children, Journal of Electromyography and Kinesiology 2(22) (2012), 259-265.

[50] Y. Uno, M. Kawato and R. Suzuki, Formation and control of optimal trajectory in human multijoint arm movement. Minimum torque-change model, Biological cybernetics 2(61) (1989), 89-101

[51] P. Vanvliet and A.M. Wing, A new challange - Robotics in the rehabilitation of the neurologically motor impaired, Physical Therapy 1(71) (1991), 39-47.

[52] C.M. Waugh, A.J. Blazevich, F. Fath and T. Korff, Age-related changes in mechanical properties of the Achilles tendon, Jour nal of Anatomy 2(220) (2012), 144-155.

[53] A.P.H. Weightman, N. Preston, R. Holt, M. Allsop, M. Levesley and B. Bhakta, Engaging children in healthcare technology design: Developing rehabilitation technology for children with cerebral palsy, Journal of Engineering Design 5(21) (2010), 579-600.

[54] D.A. Winter, Biomechanics of human movement, ed. Wiley, 1979.

[55] G. Wu, F.C.T. van der Helm, H.E.J. Veeger, M. Makhsous, P. Van Roy, C. Anglin, J. Nagels, A.R. Karduna, K. McQuade, X.G. Wang, F.W. Werner and B. Buchholz, ISB recommendation on definitions of joint coordinate systems of various joints for the reporting of human joint motion - Part II: Shoulder, elbow, wrist and hand, Journal of Biomechanics 5(38) (2005), 981-992.

[56] V. Yadav, J.P. Schmiedeler, S. McDowell and L. WorthenChaudhari, Quantifying age-related differences in human reaching while interacting with a rehabilitation robotic device, Applied Bionics and Biomechanics 4(7) (2010), 289-299. 

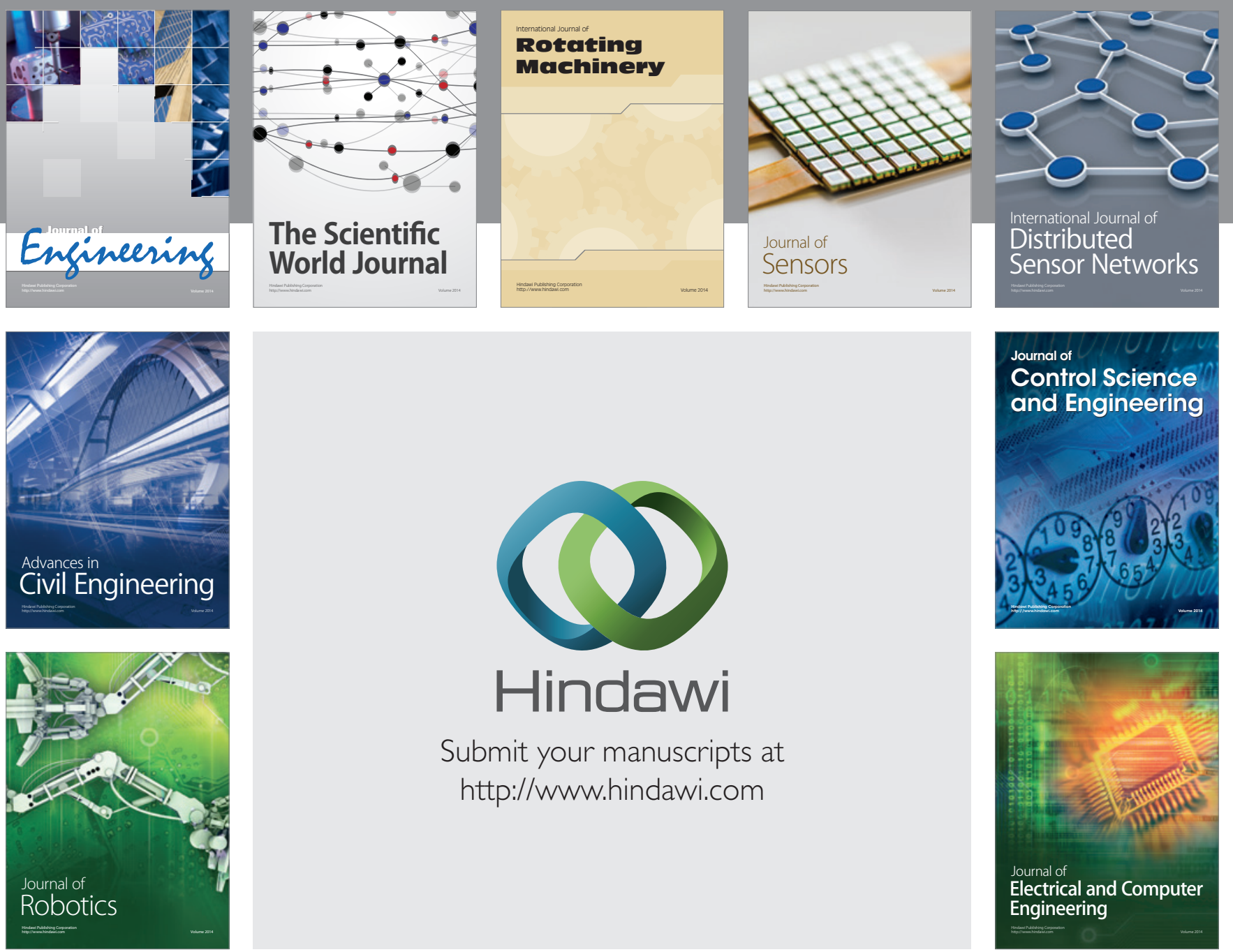

Submit your manuscripts at

http://www.hindawi.com
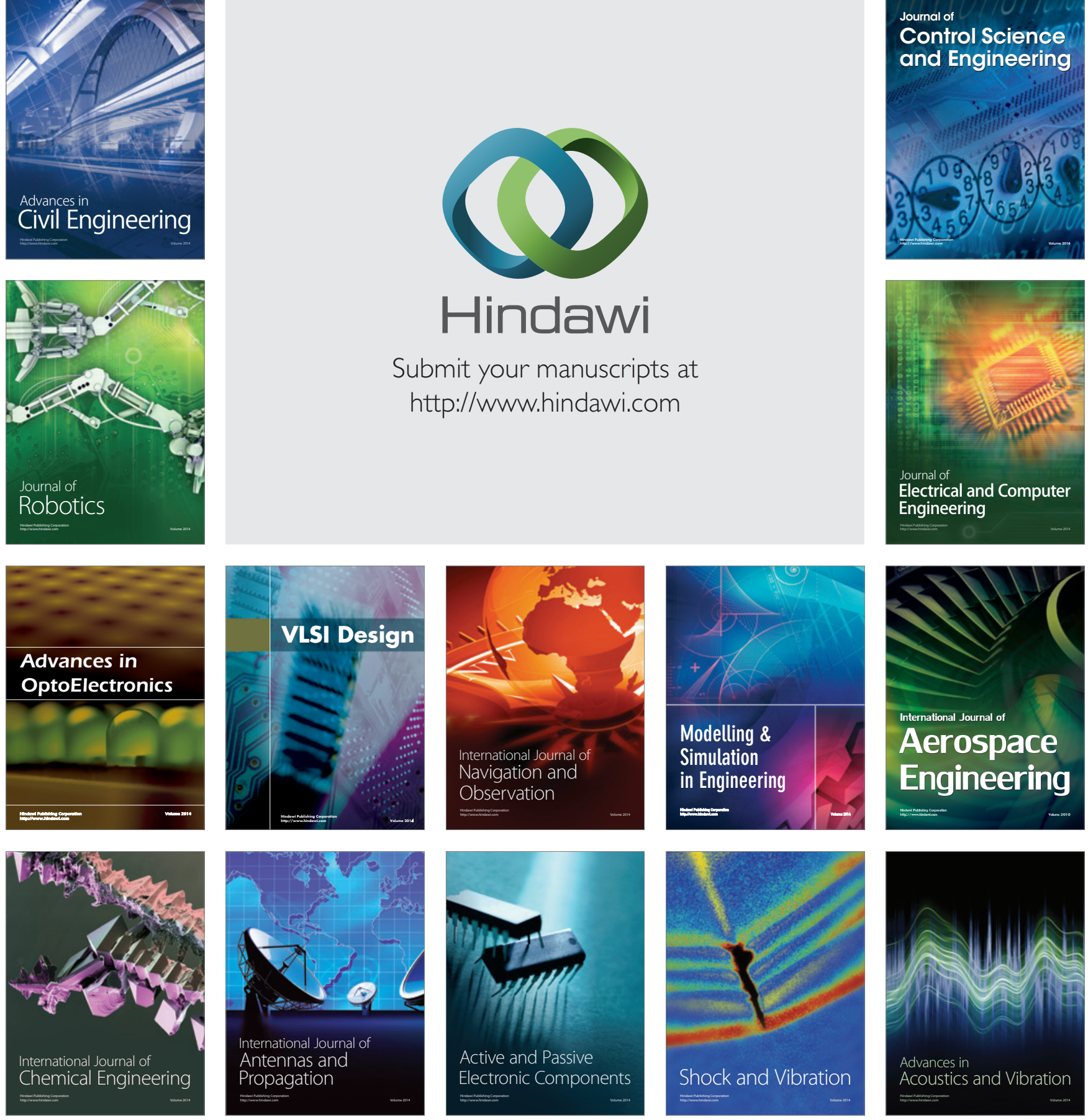